\section{Acute dystonia in a patient with 22q11.2 deletion syn- drome}

\section{Konstantinos Kontoangelos,1,2 \\ Antonis Maillis,1,2 Maria Maltezou, ${ }^{3}$ \\ Sofia Tsiori, 1 \\ Charalambos C. Papageorgiou1,2}

1First Department of Psychiatry, Eginition

Hospital, Athens University Medical

School; 2University Research Institute

of Mental Health, Athens; ${ }^{3}$ Department

of Neurology, General Oncology Hospital, Agioi Anargyroi, Greece

\section{Abstract}

The 22q11.2 deletion syndrome (di George syndrome) is one of the most prevalent genetic disorders. The clinical features of the syndrome are distinct facial appearance, velopharyngeal insufficiency, conotruncal heart disease, parathyroid and immune dysfunction however, little is known about possible neurodegenerative diseases. We describe the case of an 18-year old patient suffering from $22 q 11.2$ deletion syndrome. Since adolescence, he presented with behavioral disorders, recommended treatment with $2 \mathrm{mg}$ aloperidin and he presented cervical dystonia and emergence of torticollis and trunk dystonia. Antipsychotic medications either accelerate or reveal dystonic symptoms.

\section{Introduction}

The $22 q 11.2$ deletion syndrome is one of the most prevalent genetic disorders and has been found to be one of the most common causes of learning disability and mild mental retardation. ${ }^{1}$ It is caused by micro deletion in the long arm of chromosome 22 and its clinical characteristics include congenital heart disease; palatal abnormalities; hypocalcaemia and Tcell immunodeficiency; and abnormal faces that include a bulbous nasal tip and prominent nasal root, narrow and with flat cheeks, narrow palpebral fissures, small mouth, receding chin and small cupped ears. ${ }^{2}$ Children with $22 q 11.2$ deletion syndrome have been found to have a substantially greater risk for the development of schizophrenia and bipolar disorder with approximately 25-30\% presenting psychotic symptoms in adolescence. ${ }^{3}$

\section{Case Report}

We describe the case of an 18-year old patient suffering from $22 q 11.2$ deletion syndrome. Since adolescence, he manifested with behavioral disorders, aggression, verbal abuse and sleep disorders. Diagnosis of $22 q 11.2$ was confirmed at the age of 3 with a special chromosomal detector (D22 s75, ONCOR). His performance at school was poor. After a heated argument with his parents, he presented intense anger, started breaking and throwing things and was verbally aggressive. His parents contacted the treating physician, who recommended treatment with $2 \mathrm{mg}$ aloperidin. The patient received treatment dissolved in water. The treatment calmed him down, but a few hours later, he presented with cervical dystonia and emergence of torticollis and trunk dystonia with continuous twisting movements of the trunk and limbs, as well as accompanying dysarthria and pain. The patient visited the emergency outpatient clinic of the hospital, where he was given 1 amp Biperiden i.m. His acute dystonia was considered a side effect of aloperidin administration and he was subsequently subjected to full laboratory testing to investigate the risk factors associated with acute dystonia. The brain magnetic resonance imaging (MRI) revealed no abnormal findings and he was then subjected to a DATscan to investigate a possible dysfunction of the basal ganglia, which proved negative. After two weeks the patient was hospitalized in a psychiatric clinic and was administered quetiapine $200 \mathrm{mg}$, p.o. This particular treatment improved his clinical picture, without manifesting the usually induced side effects evoked by haloperidol.

\section{Discussion}

A literature search was conducted using the Medline computer database. It focused on all studies concerning $22 q 11.2$ deletion syndrome and dystonia. The keywords were selected from titles, abstract and keywords and they were the following: Dystonia parkinsonism and $d i$ George syndrome. Butcher et al. ${ }^{4}$ suggest that $22 q 11.2$ deletions represent a novel genetic risk factor for early-onset of Parkinson disease (PD) with variable neuropathological presentation reminiscent of LRRK2-associated PD neuropathology. Shulman et al..$^{5}$ emphasize that the 22q11.2DS-associated microdeletion affects approximately 30 genes, including several intriguing candidates with potential links to PD pathophysiology. Among the genes in this region is COMT, encoding a key enzyme involved in dopamine catabolism and an established therapeutic target in PD. Zaleski et al. ${ }^{6}$
Correspondence: Konstantinos Kontoangelos, Athens University Medical School, First Department of Psychiatry, Eginition Hospital, 74 Vas Sofias Avenue, 11528, Athens, Greece,

Tel.: +30.210.728.9189 - Fax: +30.210 .724 .2020 .

E-mail: kontange@hol.gr

Key words: Velocardiofacial syndrome; 22q11 deletion syndrome; psychosis; neuropsychology; dystonia.

Contributions: KK designed the study and wrote the first draft of the manuscript; AM and author MM also contributed to the first draft of the manuscript; ST managed the literature searches of the manuscript; CCP had the overall supervision and corrected the final draft.

Conflict of interest: the authors declare no potential conflict of interest.

Received for publication: 14 March 2015.

Revision received: 8 April 2015

Accepted for publication: 15 April 2015.

This work is licensed under a Creative Commons Attribution NonCommercial 3.0 License (CC BYNC 3.0).

(C) Copyright K. Kontoangelos et al., 2015

Licensee PAGEPress, Italy

Mental Illness 2015; 7:5902

doi:10.4081/mi.2015.5902

suggest that the genetic of PD is complex. An increased risk to first degree relatives, even in apparently sporadic cases, suggests multifactorial causation of PD. Hereditary forms account for only about $1-3 \%$ of all PD cases and tend to have younger age at onset. Mutations in parkin, PINK1 and DJ1 are known to cause autosomal recessive early onset $\mathrm{PD}$ and mutations in alpha synuclein, ubiquitin $\mathrm{C}$ terminal hydrolase and LRRK2 can cause autosomal dominant PD with variable age of onset. Pinquier et al. ${ }^{7}$ report a case of $22 q 11$ deletion in a 17-year-old girl that was initially diagnosed as paranoid schizophrenia. In the case describe, transitory hypocalcemia induced dystonic symptoms that were believed to be catatonic symptoms or neuroleptic secondary effects. Verri et al. ${ }^{8}$ describe a new male patient, 33-year-old, with 10p partial deletion affected by hypocalcemia, basal ganglia calcifications and a severe autistic syndrome associated with mental retardation. The patient presented with dysmorphic features and at neurological evaluation, bilateral complete cataract, hypotonia, symptoms have been observed in $14 \%$ to $28 \%$ of children with di George syndrome and the receipt of antipsychotic treatment increase the risk of dystonic symptoms. ${ }^{9}$ Dystonia induced by aloperidin may arise from an imbalance between dopaminergic and mus- 
carinic receptors activity in nigrostriatum in the brain. ${ }^{10}$ The possible side effects of antipsychotic medications - either accelerating or reveal dystonic symptoms - merit further study.

\section{Conclusions}

Studies of di George syndrome add to the global understanding of the pathophysiology of the neurological symptoms of this common neurodegenerative disease.

\section{References}

1. Lajiness-0'Neil R, Beaulieu I, Asamoah A, et al. The neuropsychological phenotype of velocardiofacial syndrome (VCFS): rela- tionship to psychopathology. Arch Clin Neuropsychol 2006;21:175-84.

2. Gothelf D, Lombroso PJ. Genetics of childhood disorders: XXV. Velocardiofacial syndrome. J Am Acad Child Adolesc Psychiatry 2001;40:489-91.

3. Eliez S, Antonarakis SE, Morris MA, et al. Prenatal origin of the deletion $22 q 11.2$ and brain development in velocardiofacial syndrome. Arch Gen Psychiatry 2001;58:64-8.

4. Butcher NJ, Kiehl TR, Hazrati LN, et al. Association between early-onset Parkinson disease and 22q11.2 deletion syndrome: identification of a novel form of Parkinson disease and its clinical implications. JAMA Neurol 2013;1:1359-66.

5. Shulman JM. Structural variation and the expanding genomic architecture of Parkinson disease. JAMA Neurol 2013; 70:1355-6.

6. Zaleski C, Bassett AS, Tam K, et al. The cooccurrence of early onset Parkinson dis- ease and $22 \mathrm{q} 1.2$ deletion syndrome. Am J Med Genet A 2009;149:525-8

7. Pinquier C, Héron D, de Carvalho W, et al. Microdeletion 22q11: apropos of case of schizophrenia in an adolescent. Encephale 2001:27:45-50.

8. Verri A, Maraschio P, Devriendt K, et al. Chromosome 10p deletion in a patient with hypoparathyroidism, severe mental retardation, autism and basal ganglia calcifications. Ann Genet 2004;47:281-7.

9. Jolin EM, Weller RA, Weller EB. Psychosis in children with velocardiofacial syndrome (22q11.2 deletion syndrome). Curr Psychiatry Rep 2009;11:99-105.

10. Matsui-Sakata A, Ohtani H, Sawada Y. Pharmacokinetic-pharmacodynamic analysis of antipsychotics-induced extrapyramidal symptoms based on receptor occupancy theory incorporating endogenous dopamine release. Drug Metab Pharmacokinet 2005;20:187-99. 